\title{
La traduction au service de la politique. Le succès de Jacques Necker dans les lumières espagnoles
}

Jesús Astigarraga

\section{(2) OpenEdition \\ 1 Journals}

\section{Édition électronique}

URL : https://journals.openedition.org/ahrf/12005

DOI : $10.4000 /$ ahrf. 12005

ISSN : 1952-403X

Éditeur :

Armand Colin, Société des études robespierristes

\section{Édition imprimée}

Date de publication : 1 juin 2011

Pagination : 3-27

ISSN : 0003-4436

\section{Référence électronique}

Jesús Astigarraga, «La traduction au service de la politique. Le succès de Jacques Necker dans les lumières espagnoles », Annales historiques de la Révolution française [En ligne], 364 | avril-juin 2011, mis en ligne le 01 juin 2014, consulté le 23 avril 2022. URL : http://journals.openedition.org/ahrf/12005 ; DOI : https://doi.org/10.4000/ahrf.12005 
A R T I C L E S

\title{
LA TRADUCTION AU SERVICE DE LA POLITIQUE. LE SUCCÈS DE JACQUES NECKER DANS LES LUMIÈRES ESPAGNOLES
}

\author{
Jesús ASTIGARRAGA
}

\begin{abstract}
Cet article analyse la réception de l'œuvre de Jacques Necker dans l'Espagne des Lumières. Il étudie en détail les différentes traductions dont celle-ci fit l'objet et la façon dont les Espagnols des Lumières utilisèrent les idées de Necker dans différents domaines de la politique économique, principalement les finances publiques, le commerce des grains et l'administration territoriale. II démontre ainsi que les deux aspects (traduction et influence) sont étroitement liés et que l'importance de la circulation de ces idées dans l'Espagne de la fin du xviiie siècle est largement due à leur utilisation politique.
\end{abstract}

Mots-clés : histoire de la pensée économique, circulation internationale des idées, Lumières espagnoles, finances publiques, commerce des grains, administration territoriale.

« La circulation des idées est, de tous les genres de commerce, celui dont les avantages sont les plus certains. [...] c'est à l'universel qu'il faut tendre, lorsqu' on veut faire du bien aux hommes [...] Traduire un poète, ce n'est pas prendre un compas et copier les dimensions de l'édifice; c'est animer du même souffle de vie un instrument différent », écrivait en 1816 Madame de Staël ${ }^{1}$. Ce judicieux commentaire reflète à merveille l'esprit d'une époque que l'auteur vivait aux premières loges, et dont les bienfaits, au moment où elle écrivait, devaient lui apparaître évidents. Même

(1) Anne-Louis Germaine Necker, Baronne de StaËL-Holstein, De l'esprit des traductions (1816), Euvres inédites de Mme la Baronne de Staël publiées par son fils, Paris, 1821, tome III, p. 345 . 
s'il manque encore une synthèse sur l'imposant héritage du XVIII ${ }^{\mathrm{e}}$ siècle en matière de traductions, il est indéniable que, sans celles-ci, il serait difficile d'expliquer la nature cosmopolite des Lumières et la bénéfique circulation des idées à laquelle fait allusion Madame de Staël. Quoi qu'il en soit, on peut mieux comprendre la justesse de son appréciation si l'on considère qu'elle fut directement témoin de l'énorme succès de traduction que connut l'œuvre de son père, Jacques Necker. Comme cela a été largement démontré, celui-ci fut en effet l'auteur de quelques-uns des principaux best-sellers du XVIII' siècle dans le domaine de l'économie politique ${ }^{2}$.

Bien qu'on ne dispose pas d'une analyse définitive de cet énorme succès européen, il ne fait aucun doute que l'œuvre de Necker atteignit pleinement l'Espagne ${ }^{3}$. L'illustre Genevois y était bien connu, non seulement comme auteur d'une œuvre importante mais aussi comme personnalité politique d'envergure européenne, notamment durant son premier passage au ministère des Finances (1776-1781). Ces deux dimensions de la personnalité de Necker étaient très présentes en Espagne durant les vingt dernières années $d u \mathrm{XVIII}^{\mathrm{e}}$ siècle : tandis que son parcours politique était abondamment commenté dans la presse - notamment dans les deux journaux officiels de l'époque, le Mercurio histórico y político et la Gaceta de Madrid ${ }^{4}$-, son œuvre connaissait une grande diffusion dans toute la péninsule. De fait, Necker fut l'un des économistes européens les plus traduits en Espagne au XVIII ${ }^{\mathrm{e}}$ siècle : les différentes versions de ses textes embrassent quatre de ses principaux écrits : Sur la législation et le commerce des grains (1775); Compte rendu au Roi (1781); Mémoire de M. Necker au Roi sur l'établissement des administrations provinciales

(2) Kenneth E. CARPENTER, « The Economic Bestsellers before 1850 », Bulletin of the Kress Library of Bussines and Economics, 1975, $\mathrm{n}^{\circ} 11$, p. 22-23. Les nombreuses allusions faites au cours de ce travail à Necker et ses écrits proviennent des travaux suivants : Henri Grange, Les idées de Necker, Paris, 1974; Jean Egret, Necker, Ministre de Louis XVI (1776-1790), Paris, 1975 ; Robert D. Harris, Necker. Reform Statesman of the Ancien Régime, Berkeley/Los Angeles/Londres, 1979; Id., Necker and the Revolution of 1789, Londres/Boston, 1986; Franco VENTURI, Settecento riformatore. La cadutta dell'Antico Regime (1776-1789), Torino, 1984, vol. IV, p. 329 sqq.

(3) Le présent article complète et reformule sur la base de nouveaux arguments les premières interprétations que nous avions proposées il y a quelques années dans « La obra de Jacques Necker : una influencia fundamental en la Ilustración económica española », dans Enrique FuENTES Quintana (ed.), Economía y economistas españoles. Vol. III : La Ilustración, Barcelone, 2000, p. 729-753, et dans « Necker en España, 1780-1800», Revista de Economía Aplicada, 2000, n 23, p. 119-141.

(4) Ce ton de louange envers Necker se retrouve aussi dans un bref manuscrit destiné à expliquer les mesures de son premier ministère et les causes de sa démission : Monsieur Necker, Director General de Real Hacienda en Francia. Su retiro del Ministerio, el año de 1781 (Biblioteca Nacional, Madrid, nº 12939-17, 16 ff., c. 1781). 
(1781) et De l'Administration des finances de la France (1784) $)^{5}$. Par ailleurs, ces versions espagnoles possèdent toutes un caractère politique marqué : élaborées grâce à la protection de puissants personnages, elles devaient servir à la conception des réformes entreprises sous les règnes de Charles III et Charles IV. En ce qui concerne l'Espagne, Necker constitue ainsi un cas relativement paradigmatique d'auteur dont la diffusion s'explique par un contexte de circulation des idées étroitement lié à des motifs d'ordre politique. De fait, il s'agit certainement d'un auteur crucial si l'on veut comprendre la pensée et les réformes qu'inspirèrent les Lumières espagnoles à la fin du XVIII ${ }^{\mathrm{e}}$ siècle, en particulier dans les trois domaines qui feront l'objet de cette analyse : les finances publiques, le commerce des grains et l'administration du territoire.

\section{Les finances publiques}

Le premier ouvrage de Necker traduit en Espagne est le Compte rendu. Publié à Paris en janvier 1781, ce livre constituait l'un des actes les plus décisifs de son premier ministère : il s'inscrivait dans une « stratégie d'ensemble » qui comprenait des motifs financiers, l'appel à l'opinion publique et la justification de la trajectoire politique de son auteur ${ }^{6}$. Si 1'Espagne ne resta pas en marge de l'aire d'influence du Compte rendu, le succès espagnol de l'ouvrage pose certains problèmes d'interprétation. Trois traductions de l'ouvrage furent probablement réalisées en Espagne au cours de l'année 1781 mais seule l'une d'entre elles vit le jour, sous la forme de huit livraisons publiées anonymement dans le Mercurio histórico y político.

La première de ces traductions, dont on n'a plus de traces à l'heure actuelle, est probablement due à Miguel del Castillo. Ce prêtre résidant à Madrid, spécialiste de traductions religieuses, adressa en janvier 1781 une requête au Conseil de Castille dans le but de publier sous forme de livre une version du Compte rendu. En mars 1781, cette requête obtint 1'appro-

(5) Dans la suite du texte, nous désignerons ces ouvrages de la manière suivante : Sur la législation, Compte rendu, Des Administrations Proviciales et Des Finances. En revanche, il n'existe que des preuves lacunaires de la circulation en Espagne des deux premiers écrits économiques de Necker, son bref feuillet de 1769 en faveur de la Compagnie des Indes Orientales et l'Éloge de JeanBaptiste Colbert (1773).

(6) Léonard Burnand, « Préface » à J. Necker, Compte rendu au Roi (1781), Genève, 2005, p. VIII-XIII. Durant la seule année 1781, cinq éditions parurent en France, ainsi que diverses traductions en anglais, en danois, en italien, en allemand et en espagnol, faisant ainsi de l'ouvrage l'un des livres les plus vendus au XVIII ${ }^{\mathrm{e}}$ siècle sur la question des finances. 
bation des censeurs de l'Académie d'histoire, Guevara Vasconcelos et Capmany. Ceux-ci estimaient que l'œuvre de Necker était « excellente » et contenait de « très grands principes d'économie politique » mais ils la considéraient comme " extrêmement difficile à traduire, tant à cause de son objet qu'en raison du succès de son auteur $\gg^{7}$. Cela étant, ils reconnaissaient la qualité de la version de Castillo et le félicitaient d'avoir introduit une longue note destinée à corriger l'unique défaut qu'ils trouvaient au texte original : l'opposition de Necker au système du " papier monnaie ", que le Genevois paraissait assimiler à l'échec du système financier de Law. Ce jugement était certainement à même de causer du tort à la récente décision des autorités financières espagnoles d'émettre des titres de la dette publique (vales reales). L'affaire était d'importance : à la même époque, l'influent Cabarrús écrivait, de Paris, au Fiscal Campomanes, pour tenter d'éviter une publication officielle du "Compte rendu de M. Necker», notamment en raison de sa critique du « papier monnaie $»^{8}$. Quoi qu'il en soit, au moment où les membres de l'Académie d'histoire rendaient leur jugement, la seconde traduction espagnole du Compte rendu avait déjà commencé à paraître dans le Mercurio. Guevara Vasconcelos et Capmany continuèrent néanmoins à considérer que la publication de la version de Castillo était nécessaire car elle présentait l'avantage de constituer une unité sous forme de livre9. En octobre 1781, ils firent connaître leur décision à Floridablanca, entre les mains duquel la traduction demeura.

La raison de cette obstruction est que, comme nous l'avons dit, depuis mars 1781, le Mercurio publiait sous forme de livraisons mensuelles une traduction de l'œuvre de Necker. Cette version, la deuxième de notre classement ${ }^{10}$, était dotée d'une importante charge politique.

(7) Archivo de la Real Academia de la Historia (Madrid), 11-1-4-8017-50.

(8) Néanmoins, Cabarrus, l'opposant le plus actif à la circulation de l'œuvre de Necker en Espagne, ne jugeait pas plus positivement son Compte rendu auprès de Campomanes : « Le lieu et le temps ne permettent pas de m'étendre sur les défauts de cet ouvrage, écrit de manière artificieuse, combattu ouvertement ici [à Paris] et dont j'ai en ma possessions les attaques imprimées » (Archivo de la Federación Universitaria Española (Madrid), leg. 48-19).

(9) Archivo Histórico Nacional (Madrid), Consejos, leg. 5546-29.

(10) «Cuenta dada al Rey por Mr. Necker, Ministro de Hacienda », Mercurio histórico y politico, 1781, mars, p. 224-9; avril, p. 335-345; mai, p. 35-40; juillet, p. 247-253; août, p. 352-359; octobre, p. 145-152; novembre, p. 243-248; décembre, p. 338-346. Le Compte rendu fit l'objet de deux recensions dans la presse espagnole de l'époque : Correo literario de la Europa, 6 septembre 1781, p. 215-216; Mercurio histórico y político, mars 1781, p. 224. Un autre résumé très synthétique du Compte rendu se trouve dans la traduction de Eduardo duc d'Almodóvar (publié sous le pseudonyme de Eduardo Luque de Malo), «Apéndice sobre el estado político-económico de la Francia », Guillaume-Thomas RAYNAL, Historia política de los establecimientos ultramarinos de las naciones europeas, Madrid, 1784-1790, vol. III, lib. IV, p. 129-132. 
Quoiqu'anonyme, elle avait probablement été réalisée sur ordre du secrétaire d'État, le comte de Floridablanca. Le Mercurio dépendait en effet du secrétariat d'État et étant donné sa grande diffusion, ce mode de publication garantissait à cette version une circulation très large dans l'espace ibérique. Cependant, cette traduction ne reflétait pas de manière fidèle le contenu du texte original. Désordonnée, fragmentaire et surtout très incomplète, elle se bornait à rendre compte de « l'état actuel des finances de France », c'est-à-dire qu'elle reprenait la première partie du Compte rendu, mais omettait les tableaux généraux sur les ressources et les dépenses, plusieurs chapitres de la deuxième section, ainsi que toute la troisième et dernière section qui traitait des projets de réforme prévus par Necker. L'explication la plus plausible de ce traitement est qu'il répondait à des motifs politiques de première importance : si, au secrétariat d'État, on jugeait favorablement la politique « neckerienne » de transparence financière devant l'opinion publique - politique que le ministère des Finances allait adopter quelques années plus tard, sous Lerena -, il convenait à l'inverse de limiter les informations sur les projets de réforme que Necker avait imaginés pour la France, jugés trop audacieux du point de vue espagnol. Peut-être est-ce là la raison pour laquelle Floridablanca avait bloqué la traduction précédente, celle de Castillo.

La version du Mercurio n'avait probablement rien à voir avec la troisième traduction espagnole du Compte rendu, également anonyme, qui dut être réalisée au même moment et demeura, à l'époque, sous forme manuscrite ${ }^{11}$. À la différence des deux précédentes, cette nouvelle version était pratiquement complète. Elle fut réalisée d'abord par un seul auteur et révisée ensuite par (probablement) deux autres personnes : toutes les pages du manuscrit comportent de nombreuses corrections destinées à améliorer la qualité de la traduction originelle. Cette troisième traduction ne paraissait donc pas sur le point d'être publiée : c'était probablement un brouillon réalisé par plusieurs traducteurs et correcteurs. En tout cas, il s'agissait d'une version presque intégrale du texte de Necker : pratiquement dépourvue de censure, elle n'omettait que les derniers paragraphes de la troisième partie, ainsi que les tableaux sur les ressources et les dépenses publiques figurant en annexe.

Par ailleurs, la superposition des différentes écritures dans le manuscrit montre bien les problèmes que posait la traduction d'un livre

(11) «Informe presentado a S. M. Cristianísima en el mes de Enero de 1781 sobre el estado de la Real Hacienda. Por Necker Director general de ella » (Biblioteca Nacional, $\mathrm{n}^{\circ}$ 11.185, 144 ff., c. 1781). 
comme celui de Necker, en raison de son abondante terminologie comptable et financière. De fait, l'un des auteurs avait ajouté dans la marge de nombreuses notes personnelles, destinées à éclairer divers concepts fiscaux français (corvées, aides, etc. ${ }^{12}$ et à situer les réformes de Necker par rapport à celles de Turgot, ou les idées du Compte rendu dans la perspective des travaux antérieurs du Genevois ${ }^{13}$. Un autre indice nous incite à croire qu'il pourrait également s'agir d'une traduction réalisée dans les cercles officiels, très proches du pouvoir royal : la seule partie importante du texte original qui ait été supprimée correspond aux passages dans lesquels Necker aborde les différentes objections faites à son projet des administrations provinciales, notamment celles se rapportant à leurs compétences et à leur structure de représentation. Ces considérations critiques devaient être malvenues dans le contexte espagnol alors que, comme nous le verrons, les ministres Múzquiz et Floridablanca envisageaient à la même époque de promouvoir des «Juntes provinciales pour les affaires de Finances », institutions décentralisées probablement inspirées des administrations provinciales de Necker. En conclusion, compte tenu de l'intense circulation que connut le Compte rendu en Espagne, il est remarquable que la seule version publiée de ce texte ait été incomplète, désordonnée et fragmentaire, en raison des intérêts politiques du moment.

Des Finances connut en Espagne un sort pire encore que celui du Compte rendu. Cette ouvre de maturité de Necker fit pourtant l'objet d'une tentative très sérieuse de traduction. Son auteur était le Français Thévin, éditeur et libraire résidant à Madrid, très bien placé sur le marché éditorial espagnol. En même temps qu'il obtenait le permis de vente de Des Finances en Espagne en 1785, il en commençait la traduction et entamait les démarches pour qu'elle soit approuvée par le Conseil de Castille. Cependant, une erreur inexplicable des censeurs du Conseil - qui la confondirent avec le Compte rend $u^{14}$ - suivie de la mort de Thévin, en 1787, retardèrent sa publication, et ce alors qu'apparemment il existait déjà, depuis mai 1785, une traduction du premier volume de l'ouvrage, enrichie « de notes pour sa compréhension des termes les plus obscurs et les

(12) Ibid., ff. 38, 43v, 75v, 83 et $98 \mathrm{v}$.

(13) Ibid., ff. $130 \mathrm{v}$ et 137.

(14) « Informe de M. de Lardizábal y R. de Guevara », du 29 juin 1785 (Archivo Histórico Nacional, Consejos, leg. 5541-43). Les censeurs demandaient à nouveau que soit supprimée l'allusion négative de Necker aux vales reales espagnols. Mais, à côté de cela, ils considéraient que l'ouvrage contenait « des observations très profitables et applicables même à d'autres pays sur les principaux points de l'Économie Politique et Publique ». 
plus difficiles $»^{15}$. Néanmoins, après cette date, bien que les demandes en faveur d'une traduction de Des Finances se soient multipliées, la panique suscitée dans le gouvernement espagnol par le triomphe de la Révolution française empêcha la réalisation de celle-ci, ainsi que celle d'un autre ouvrage non moins révélateur de la pensée de Necker, De l'importance des opinions religieuses $(1788)^{16}$.

De ce fait, l'Espagne demeura en marge de la remarquable diffusion internationale qu'allait connaître, par le biais de la traduction, Des Finances ${ }^{17}$. Ceci n'empêcha pourtant pas cette œuvre d'être abondamment consultée et utilisée en Espagne. De même, il faut mentionner la circulation de l'ouvrage par des voies parallèles : la preuve la plus éloquente en fut le résumé réalisé en 1786 par le duc d'Almodóvar, sous le titre d'Appendice sur l'état politique et économique de la France ${ }^{18}$. Cette version, quoique très brève, avait une importante portée politique; c'est la raison pour laquelle elle fut insérée dans la traduction espagnole de L'Histoire philosophique et politique (1770) de Raynal. Elle s'inscrivait ainsi dans une opération longuement mûrie de censure et de réélaboration méticuleuse de cette œuve polémique, dans le but de l'adapter au contexte espagnol ${ }^{19}$. L'intention de ce prestigieux diplomate, qui avait dû connaître personnellement Necker, était sans doute d'atténuer la forte composante républicaine de Raynal et de réorienter le lecteur espagnol vers un modèle politique et économique plus modéré, assimilable aux positions monarchiques et philo-britanniques caractéristiques de Necker. De fait, Almodóvar incluait également dans sa traduction de longs passages destinés à expliquer à ses lecteurs la " Constitution anglaise ».

Tandis que tout cela se produisait sur le plan éditorial, l'application des idées de Necker en matière de finances était réalisée par Pedro de Lerena, ministre des Finances de 1785 à 1791, aidé de son collaborateur

(15) Archivo Histórico Nacional, Consejos, leg. 5546-29.

(16) Voir Marcelin Defourneaux, Inquisición y censura de libros en la España del siglo XVIII, Madrid, 1973, p. 154. L'Espíritu de los mejores diarios (17-19 avril 1788, p. 51-3 et 57-8, respectivement) publia un compte rendu élogieux de cet ouvrage de Necker.

(17) Durant les trois années qui suivirent sa publication en France et en Suisse, ce troisième best seller de Necker fut traduit en anglais, en allemand, en italien, en russe et en danois. Pour le contexte français, Perrot le situe pami les livres ayant connu la plus grande diffusion de tout le $\mathrm{XVIII}^{\mathrm{e}}$ siècle, au même titre que ceux de Vauban ou de Mirabeau (Une histoire intellectuelle de l'économie politique, Paris, 1992, p. 144).

(18) Duc D’Almodovar, op. cit., vol. III (1786), livre IV. Un compte rendu élogieux de Des Finances fut publié dans la Gaceta de Madrid (25 janvier 1785, p. 52-3; 8 février 1785, p. 83-84; 4 mars 1785 , p. 144 ; 7 juin 1785, p. 360).

(19) Voir Ovidio Garcia, "Ilustración » e intereses estamentales, Madrid, 1982. 
le plus proche, Vicente Alcalá ${ }^{20}$. Des Finances constitua l'une des sources d'inspiration de sa réforme des rentas provinciales ${ }^{21}$, la plus importante de son mandat et l'une des innovations fiscales les plus marquantes de tout le $\mathrm{XVIII}^{\mathrm{e}}$ siècle espagnol. Cette réforme tentait d'apporter une solution à la grave crise financière que connaissait la monarchie depuis 1780 . Elle constituait en outre un tournant décisif dans la politique fiscale, dans la mesure où elle impliquait de renoncer aux anciens projets de certains représentants des Lumières (Zavala, Loynaz, etc.) qui envisageaient de remplacer les rentas provinciales par une « contribution unique ». Deux ministres, Ensenada (1745-1749) et Múzquiz (1766-1785), avaient déjà tenté, sans succès, de procéder à cette substitution. Le projet connut une nouvelle impulsion en 1783-1784 lorsque Cabarrús, avec l'appui de Múzquiz, présenta à Floridablanca une proposition radicale, consistant à asseoir le système fiscal sur un impôt direct portant sur la valeur des terres et des immeubles. Loin d'y consentir, Floridablanca choisit alors une voie beaucoup plus modérée : il encouragea Lerena à mener à bien la réforme des rentas provinciales dans les années 1785-1787. Celle-ci consistait en une révision systématique de l'assiette de l'impôt et en une réduction des types d'impôts théoriques payés au titre des principales rentas provinciales; de surcroît, elle établissait un nouvel impôt sur les frutos civiles, qui portait sur les affermages des terres et des autres propriétés.

La réforme de Lerena nous confronte à un cas intéressant, celui d'une utilisation active des idées de Necker pour réactiver un vieil idéal de réforme fiscale. Le principal inspirateur de celle-ci fut Alcalá, qui se montrait capable de concilier l'autorité de Necker avec les exigences de plusieurs économistes politiques espagnols des XVII ${ }^{\mathrm{e}}$ et XVIII ${ }^{\mathrm{e}}$ siècles, favorables à une réforme substantielle des rentas provinciales (Mata, Osorio, Moncada, Ulloa, Campomanes et Arriquíbar) ${ }^{22}$. La justification de cette

(20) Lerena connaissait à fond Des Finances. Voir son Mémoire rédigé en 1789 et publié par José CANGa Argüelles, Diccionario de Hacienda con aplicación a España (1834), Madrid, 1968, vol. II, p. 129-145. Il en existe également une copie manuscrite (Biblioteca Nacional, $\mathrm{n}^{\circ} 11259-46$ ).

(21) Cette dénomination recouvre un vaste ensemble d'impôts indirects dont les plus importants sont les alcabalas, les cientos et les millones, qui portent sur le commerce et la consommation, y compris des biens de première nécessité, dans les 22 provinces de la Couronne de Castille.

(22) Vicente Alcalá et Vicente Mantecón, « Perjuicios del antiguo sistema de rentas provinciales ", Continuación de las Memorias de la Real Sociedad Económica de Amigos del País de la Provincia de Segovia, Ségovie, 1787. Les deux auteurs comptaient parmi les membres dirigeants de la Société Économique de Ségovie, qui à cette époque se transformait en caisse de résonance des réformes de Lerena. Sur Alcalà, voir José Manuel VAlles, Ciencia, Economía Política e Ilustración, Madrid, 2008. 
réforme se fondait sur deux anciens principes présents dans cette tradition de pensée : l'équité des contributions et la réduction des charges fiscales portant sur les biens de consommation courante. Dans le même temps, ces principes se trouvaient validés par deux autres idées d'inspiration « neckerienne »: d'une part, l'idée selon laquelle il était plus adéquat de procéder à une réforme graduelle du système fiscal plutôt que de tenter de le « changer d'un seul coup »; de l'autre, l'avantage qu'il y avait à conserver l'impôt sur la consommation, en essayant, par conséquent, de répartir la charge totale entre les impôts directs et les impôts indirects ${ }^{23}$. Les idées de Necker n'étaient cependant pas exemptes de critiques : dans une série de lettres adressées précisément à Lerena (et non publiées à l'époque), le valencien Arroyal soulignait que la monarchie espagnole n'était plus en état d'admettre des rafistolages semblables à ceux que le " malheureux » Necker avait tenté d'appliquer en France et que la solution la plus adéquate était de procéder à une refonte intégrale de tout le système des rentes publiques $^{24}$. Toutefois, Arroyal voyait bien que l'efficacité d'un système d'organisation des finances dépendait d'autres changements fondamentaux, y compris une révision en profondeur du système politique ${ }^{25}$. Le réformisme prudent de Lerena - et de Necker - se trouvait ainsi au centre d'une controverse essentielle (changement graduel $v s$ changement radical) portant sur la stratégie à adopter pour réformer les finances de l'Espagne.

Étant donné le point de vue qu'il défendait, il était logique que Arroyal fît preuve d'un jugement sévère envers Lerena. Cependant, une vision rétrospective permet d'évaluer de façon plus nuancée l'action du ministre des Finances. De fait, il est aujourd'hui admis que, grâce à sa réforme, les finances retrouvèrent leur équilibre budgétaire, sans que le niveau de la dette publique ne s'en trouvât réduit. Ce bilan positif n'empêche pas de souligner que ses décisions constituaient une nouvelle victoire des corps privilégiés, qui demeuraient exemptés d'impôts. Elle offre également une preuve supplémentaire des obstacles que l'Ancien Régime opposait, en Espagne, à une transformation substantielle du système fiscal, laquelle était par ailleurs tout à fait nécessaire au vu des difficultés financières des vingt dernières années du XVIII ${ }^{\mathrm{e}}$ siècle. En ce sens, bien qu'il soit possible de considérer les réformes de Lerena comme une sorte de réédition de celles réalisées par Necker en France (comme

(23) Vicente Alcalá et Vicente Mantecón, op. cit., p. 63, 79, 170, 200, 201 et 204-206.

(24) León de Arroyal, Cartas político-económicas al Conde de Lerena (c. 1786-1791), Oviedo, J. Caso (éd.), 1971, p. 3, 6, 84-85 et 152.

(25) Pablo Fernández Albaladejo, Fragmentos de Monarquía, Madrid, 1992, p. 468-487. 
Arroyal lui-même le faisait remarquer à plusieurs reprises), on ne saurait en aucun cas établir de parallèle entre leurs résultats, du moins dans le sens où Boscher ${ }^{26}$ défend l'action de Necker, en la considérant comme un pas décisif pour libérer les finances royales des « intérêts privés », conformément à un critère moderne d'organisation bureaucratique.

Une influence du Compte rendu et de Des Finances peut être vue dans trois autres décisions importantes que prit Lerena au cours de son ministère. Tout d'abord, il fut le premier responsable des finances espagnoles qui s'occupa de rendre publics ses budgets. Ensuite, dans la ligne des efforts entrepris par Ensenada, Múzquiz et Floridablanca, Lerena fonda en 1786 le Bureau de la Balance de Commerce (Oficina de la Balanza de Comercio), qui fut la première institution des études empiriques sur l'économie espagnole avant d'être transformée, au début du XIX ${ }^{\mathrm{e}}$ siècle, en Département des Statistiques. Enfin, c'est sous son impulsion que fut entamé un important effort de compilation des lois de finances espagnoles. Par cette décision, Lerena tentait de répondre aux plaintes que la parution de Des Finances avait suscitées parmi les représentants des Lumières, en raison de l'absence en Espagne d'une œuvre de cette nature ${ }^{27}$. Pour mener à bien cette tâche, Lerena désigna le Fiscal Covarrubias, qui au cours de l'année 1786 avait contribué à la diffusion de Des Finances en Espagne. Le Fiscal laissa inachevé un gros travail de compilation des données historiques et législatives sur les finances espagnoles, lequel demeure inédit à ce jour ${ }^{28}$.

L'œuvre de Necker permit ainsi d'inaugurer une nouvelle étape de transparence dans les finances espagnoles. En même temps, et de la même façon qu'en France, celle-ci fut un facteur décisif de l'éclosion du nouveau concept d' " opinion publique ", à laquelle contribua également l'ouverture progressive d'une "sphère publique » au cours des années 1780 avec l'apparition d'une presse critique, de nouvelles formes de sociabilité et de la génération plus radicale des Lumières « tardives ». Y prit également part la circulation d'auteurs comme Filangieri, Turgot, ou Raynal dont les œuvres sont considérées comme décisives dans la

(26) John F. Boscher, French Finances, 1770-1795, Cambridge, 1970, p. 142-165 et 276-277.

(27) Voir, en particulier, la « Disertación sobre el Consejo de Hacienda o historia general de la administración pública », Espíritu de los mejores diarios literarios, n 212-214, décembre 1789 et janvier 1790. Faisant tacitement allusion à Necker, son auteur, D. M. M. B., y déplore que 1'Espagne soit dépourvue d'un « traité complet » sur les Finances.

(28) José Covarrubias, Código, o Recopilación de Leyes de Real Hacienda (manuscrit), Biblioteca del I. E. F. (Madrid), c. 1790. 
découverte de cette catégorie fondamentale de la discussion éclairée à la fin du XVIII ${ }^{\mathrm{e}}$ siècle, formant une espèce de " tribunal invisible, impersonnel et anonyme ${ }^{29}$. Il faut souligner, dans le cas de l'Espagne, l'énorme importance des débats économiques dans l'émergence de cette « opinion publique $»^{30}$, d'où découle la centralité de la figure de Necker : ce dernier peut être considéré comme pionnier dans l'apparition de «l'économie politique de l'opinion publique », dans la mesure où il affirme qu'il est impossible d'améliorer l'efficacité du système économique sans le contrepoids institutionnel de l'opinion publique. On sait que ce concept est inhérent à l'ensemble de son œuvre : simplement défini dans le Compte rendu comme "publicité », il fut ensuite repris, dans Des Finances et Sur le compte rendu au Roi en 1781. Nouveaux éclaircissements (1788), comme une catégorie conceptuelle plus complexe ${ }^{31}$. Dans un contexte strictement économique, celle-ci était interprétée de trois manières : l'opinion publique était d'abord comprise comme une politique de transparence sur la situation économique et financière du pays et sur les méthodes de comptabilité publique employées. En second lieu, elle était vue comme une espèce de "tribunal anonyme » ayant la compétence de juger et qui, par là même, exigeait que soit établie « une sorte d'harmonie entre l'opinion publique et l'administration des Finances $\rangle^{32}$. Enfin, elle était considérée comme un facteur institutionnel indispensable, destiné à contrôler la moralité publique des hommes politiques et des fonctionnaires, Necker allant jusqu'à présenter son ouvrage Des Finances comme l'ensemble des principes de morale et de politique essentiels pour rendre compatibles la félicité des personnes et la prospérité de l'administration ${ }^{33}$.

De la même façon qu'en France, le cadre contraignant des pratiques fiscales et des procédures arbitraires de l'administration de la monarchie se révéla décisif dans l'émergence progressive de ce tribunal du « public » (et la même chose pourrait être dite du marché des grains).

(29) Mona Ozouf, «L'opinion publique », dans Keith M. BAKer, The French Revolution and the Creation of Modern Political Culture, Oxford, 1987, 5 vol.

(30) Cette question a été mise en évidence par Keith M. Baker pour le cas de la France : «Politics and Public Opinion under the Old Regime : some reflections », dans J. R. Censer et J. D. Popkin (ed.), Press and Politics in Pre-Revolutionary France, Berkeley-Los Angeles-London, 1987.

(31) Voir Léonard BuRnAnd, Necker et l'opinion publique, Paris, 2004 et Javier FernÁNDEZ Sebastián, «Introduction », dans Javier Fernández Sebastián et Joëlle Chassin (coord.), L'avènement de l'opinion publique, Paris, 2004, p. 9-29.

(32) Jacques NeCKER, Sur le compte rendu au Roi au 1781, op. cit., p. 13.

(33) Ibid., p. 2-4. 
L'arrière-plan de cette question est la situation extrêmement grave dans laquelle se trouvaient les finances espagnoles à partir des années 1780 . Cette dernière fit l'objet d'un débat rigoureux qui connut son apogée lors des Cortes de Cádiz et puisa à plusieurs sources doctrinales (Necker, Smith, l' « impôt unique » des physiocrates, etc.). Ces échanges eurent lieu dans le cadre d'une discussion publique et ouverte, à laquelle prirent part la majorité des économistes de l'époque et, dans certains cas, dans une perspective qui prônait des solutions plus profondes, bien au-delà des bornes modérées de la réforme officielle. Fait remarquable, ces économistes étaient parfaitement au courant des débats sur les finances qui étaient menés dans la lointaine France et connaissaient, entre autres, l'ardente polémique qui opposa dans les années 1787 et 1788 Calonne et Necker sur la situation réelle des finances françaises et les responsabilités du premier ministère de Necker en la matière (le fameux Discours de Calonne devant l'assemblée des notables du 22 février 1787 fut immédiatement traduit en Espagne) ${ }^{34}$. De même, divers textes de l'époque montrent que la réponse de Necker, dans laquelle il incluait ses « nouveaux éclaircissements » de son Compte rendu (1788), était également bien connue en Espagne ${ }^{35}$. Il n'est donc guère surprenant qu'un auteur assez radical des Lumières «tardives » aussi illustre que Aguirre ait identifié en 1788 l'autorité de Necker et de son Compte rendu comme le point de départ d'une politique de transparence, qui devrait donner lieu à un audacieux programme d'édition des "papiers ministériels et publics » destinés à l'opinion publique ${ }^{36}$. C'est là un fait de plus qui montre que le personnage de Necker fut central dans le processus de modernisation des finances espagnoles dans les dernières années du XVIII ${ }^{\mathrm{e}}$ siècle. De même, l'ensemble de son œuvre sur la question participa à la formation de deux générations d'économistes et de

(34) Charles A. Calonne, Discurso con que dio principio el rey de Francia a su Asamblea de Notables tenida en 22 de febrero de 1787 y el que pronunció en su nombre y presencia en dicho día Mr. de Calonne, ministro de Hacienda... traducido del francés al castellano por D. S. R. T., Madrid, c. 1788 .

(35) Les Espagnols des Lumières découvrirent ces débats sur les finances grâce aux Annales de Linguet; voir par exemple l'Espiritu de los mejores diarios literarios (17 juillet 1788, $\mathrm{n}^{\circ} 136$, p. 129-13). Voir, dans la même publication, le texte anonyme « Estado fiel de las revoluciones de la Real Hacienda en el Reino de Francia y sus situación actual » ( $n^{\circ} 136$, juillet 1788), dans lequel étaient présentés les budgets publics de Terray, Turgot, Clugny, Necker, Fleury, d'Ormesson et Calonne, probablement extraits des récents écrits de Calonne et de Necker.

(36) Manuel de Aguirre, « Carta del Militar Ingenuo sobre el fanatismo y la ignorancia » (1788), dans Antonio Elorza (ed.), Cartas y Discursos del Militar Ingenuo al Correo de los Ciegos de Madrid, 1974, p. 331-335. 
financiers ${ }^{37}$. De fait, tous les grands auteurs du tournant du siècle, de Foronda à Cabarrús en passant par Salas, utilisèrent largement ses idées en matière de finances, lesquelles furent également très présentes dans les débats des Cortes de Cádiz ${ }^{38}$.

\section{Le commerce des grains}

Aux diverses traductions du Compte rendu fit suite celle de Sur la législation et le commerce des grains (1775). Ce livre fut le premier écrit de Necker qui lui assura un indiscutable succès public ${ }^{39}$. Au-delà des circonstances précises qui expliquent sa genèse - il fut élaboré en pleine « guerre des farines »- l'ouvrage ne peut se comprendre que dans le contexte du débat crucial et prolongé que la pensée économique européenne développa tout au long du XVIII ${ }^{\mathrm{e}}$ siècle sur le commerce des grains. Sur la législation introduisait dans le débat une conception pragmatique, réaliste et relativiste, au contenu social marqué, tendant à considérer le grain comme une marchandise particulière. C'était, enfin, une vision relativement paternaliste qui, comme l'a bien montré Steven Kaplan, devait beaucoup au « stock commun des idées sur l'administration et la "police" » et mettait au centre de l'analyse la question des responsabilités de l'administration publique dans le contrôle des subsistances et la défense des intérêts du consommateur comme aucune autre autorité de l'Ancien Régime ne l'avait fait avant lui ${ }^{40}$.

La traduction espagnole de Sur la législation fut publiée dans un périodique intitulé Memorias instructivas, y curiosas (1778-1789, 12 vol.). Celui-ci fournissait au lecteur espagnol différentes informations extraites de dictionnaires, de publications périodiques ou de mémoires de diverses académies scientifiques étrangères, en particulier celle de Paris.

(37) Une autre preuve en est qu'il fut utilisé comme texte de référence dans la Chaire d'Économie Civile de la Société Économique Aragonaise, la première du genre en Espagne. Le processus de modernisation doit également beaucoup à l'influence du ministre britannique Grenville, dont les textes furent traduits avant ceux de Necker et firent l'objet de quatre éditions entre 1770 et 1787 .

(38) Voir Fernando LóPez Castellano, Liberalismo económico y reforma fiscal, Grenade, 1995.

(39) On estime qu'en France vingt éditions furent réalisées en dix ans. Dans le même temps parurent plusieurs traductions en anglais, en allemand et en italien, avant la traduction espagnole de 1783. L'édition de Sur la législation utilisée ici est celle de Eugène DAIRE, Mélanges d'Économie Politique, Paris, 1847.

(40) Steven L. Kaplan, Bread, Politics and Political Economy in the Reign of Louis XV, La Haye, 1976, p. 3, 258-259, 506 et 677. 
Son directeur était Suárez y Núñez dont le principal emploi fut celui d'archiviste de la Junte Royale du Commerce, des Monnaies et des Mines (Real Junta General de Comercio, Monedas y Minas). Ce poste lui permit d'entreprendre un important travail de traduction, dont le résultat fit de lui l'un des traducteurs les plus prolifiques des Lumières espagnoles. De même, il faut souligner son appartenance à quelques-unes des institutions les plus représentatives des Lumières espagnoles, dont les puissantes Sociétés des Amis du Pays du Pays Basque et de Madrid. Ce fut probablement sous la protection de cette dernière qu'il publia les Memorias instructivas. Les principaux sujets de cette publication périodique étaient les arts et métiers et les sciences naturelles mais elle publiait également, quoique de manière sporadique, des mémoires économiques. Des traductions espagnoles des principaux ouvrages de Condillac, Turgot, Bigot de Sainte-Croix et Justi accompagnèrent dans différents volumes celle de Necker. Celle-ci fut publiée en 1783 dans le tome VIII; elle s'accompagnait d'une mention indiquant qu'il s'agissait de l'œuvre d'un ministre des Finances français ${ }^{41}$. Il y a peu de choses à dire quant à la qualité de cette traduction : la version espagnole était correcte et très fidèle à l'original. Suárez se contentait d'introduire quatre nouvelles notes, dans le but d'éclairer la signification de certaines unités de mesure employées dans le contexte français et de l'existence, dans l'agriculture espagnole, du système des approvisionnements publics organisés autour des halles aux grains municipales.

Le but de la traduction de Suárez était sans doute de faire connaître les positions de Necker sur la scène publique espagnole afin d'intervenir dans le débat sur le marché des grains qui se développait depuis les années 1750-1760. Ce débat, relativement bien connu, puisait à des sources doctrinales diverses, principalement françaises et italiennes : le spectre allait du libéralisme doctrinaire des physiocrates jusqu'aux positions conservatrices de la pensée administrative, en passant par ce vaste groupe d'auteurs qui occupaient des positions intermédiaires et étaient en général favorables à une grande libéralisation du commerce intérieur, mais pas extérieur. Ces dernières positions étaient les plus courantes en Espagne, comme le montre le fait que le Français Herbert fut l'auteur le plus souvent traduit (en 1755, 1765 et 1795) et celui qui influença le plus la réforme officielle libérant le commerce des grains, inaugurée par la

(41) La version, publiée sous le titre Sobre la legislación, y comercio de granos, occupe les pages 1 à 237 du volume mentionné, et les Memorias no LXXIII et LXXIV. 
Pragmática de $1765^{42}$. De son côté, la traduction de Suárez se rattachait à un courant doctrinal qui avait eu comme premiers référents Forbonnais et Galiani dont les livres avaient respectivement été traduits en 1765 et 1775. Plus encore, la demande généralisée d'une libéralisation du commerce des grains par les Espagnols des Lumières s'était nourrie, en plus des auteurs déjà mentionnés, d'un amalgame très divers d'écrits agraristes, libéraux éclectiques, agronomes ou caméralistes tardifs (Melon, le Mirabeau préphysiocrate, Goudar, Plumard de Danguel, Bielfeld, Accarias de Serionne, etc.), dont les auteurs les plus influents étaient sans doute ceux du cercle de Gournay. Au sujet du commerce des grains, ceux-ci défendaient une position moins dogmatique et plus pragmatique et prudente que celle des physiocrates ${ }^{43}$, position qui allait constituer, selon les termes de Philippe Minard, le «système intermédiaire » de Necker ${ }^{44}$. Ceci permet de comprendre pourquoi ce dernier est rapidement devenu un auteur influent dans l'Espagne de la fin du XVIII ${ }^{\mathrm{e}}$ siècle, sans que cette influence ait eu toutefois pour but, à la différence de la France, de couper court au courant physiocrate, lequel existait en Espagne mais restait minoritaire ${ }^{45}$.

Le succès des idées de Necker sur le commerce des grains est également lié à celui de sa méthode réaliste et relativiste, opposée à l'« esprit de système » propre aux «économistes » et à Turgot. Nombreux furent les Espagnols des Lumières qui rencontrèrent chez Necker - et Galiani - le fondement théorique de ces principes méthodologiques. De ce fait, il n'est pas rare de rencontrer dans la littérature économique espagnole des années 1780 et 1790 des arguments tirés de l'économiste genevois, opposés à la validité des lois économiques abstraites et générales ainsi qu'aux politiques qui en découlent. Ce rejet eut en Espagne une expression concrète dans le refus du principe de liberté absolue du commerce que défendaient les physiocrates. L'influence de ces positions atteignit même les économistes les plus enclins à utiliser des méthodes déductives, comme Foronda et Jovellanos, qui acceptèrent en partie les

(42) Vicent Llombart, Campomanes, economista y politico de Carlos III, Madrid, 1992, p. 170-171. Sur la politique espagnole des grains à cette période, voir Concepción DE CASTRO, El pan de Madrid, Madrid, 1987, p. 115-181.

(43) Cf. Catherine Larrère, L'invention de l'économie au XVIII siècle, Paris, 1992; Antoin E. Murphy, «Le développement des idées économiques en France (1750-1756)», Revue d'histoire moderne et contemporaine, $\mathrm{n}^{\circ}$ XXXIII, oct.-déc. 1986, p. 521-541.

(44) Philippe Minard, La fortune du colbertisme, Paris, 1998.

(45) Sur la physiocratie en Espagne, voir Ernest Lluch et Lluis Argemí, Agronomía y fisiocracia en España (1750-1820), Valence, 1985. De nouvelles réflexions dans Jesús AstigarRaGA et Javier Usoz, "Algunas puntualizaciones sobre la fisiocracia en la Ilustración tardía española », Revista de Historia Económica, n $^{\circ}$ XXVI, 2008, p. 489-498. 
positions méthodologiques de Necker. Enfin, la majorité des auteurs qui reconnurent l'utilité de ses méthodes se prononcèrent en faveur de l'usage de l'arithmétique politique et des techniques quantitatives pour étudier la réalité économique espagnole et, en particulier, celle du marché des grains, question très présente dans la conduite politique de Necker et dans son héritage économique ${ }^{46}$.

Ce n'est donc pas un hasard si Sur la législation fut un texte abondamment utilisé par les auteurs des Lumières dans la dernière partie du $\mathrm{XVIII}^{\mathrm{e}}$ siècle. La preuve la plus significative de cette influence se trouve dans trois adaptations-plagiats réalisées en Catalogne, en Navarre et en Aragon, avant même que la traduction espagnole ne voie le jour. Dans ces écrits étaient utilisées quelques-unes des thèses les plus importantes de Necker, que ce soit celles de nature institutionnelle (la dysharmonie des intérêts ou la conception fonctionnelle de la propriété) ou celles plus proprement économiques (l'asymétrie du pouvoir et de l'information sur les marchés, la dimension temporelle des échanges ou la tendance à fixer les salaires au minimum de subsistance) ${ }^{47}$.

Selon Ernest Lluch ${ }^{48}$, Sur la législation fut l'axe théorique qui structura le deuxième plus important texte économique élaboré en Catalogne au XVIII ${ }^{\mathrm{e}}$ siècle. Il s'agit d'un long Discurso sobre la agricultura, comercio e industria del Principado de Cataluña, anonyme et dû, dans ses passages théoriques, à l'économiste catalan Caresmar, élaboré vers 1780 à l'initiative de la Junte de Commerce de Barcelone. Le choix de Necker comme « maitre à penser » n'avait rien de fortuit : son libéralisme modéré, son programme décentralisateur et son projet de régulation du commerce des grains au bénéfice du consommateur et du développement des manufactures constituaient des propositions très proches des intérêts idéologiques et réformistes défendus par cette puissante institution catalane et les secteurs du commerce qu'elle représentait.

Dans le royaume de Navarre, des thèses extraites de Sur la législation furent utilisées tout au long des années 1780 dans des buts divers ${ }^{49}$.

(46) Jean-Claude Perrot, op. cit., p. 133 et 390.

(47) Sur ce thème, voir par exemple Philippe SteIner, « La liberté du commerce : le marché des grains », Dix-huitième siècle, n 26, 1994, p. 209-211; Gilbert FACCARELlo, «"Nil Repente!" : Galiani and Necker on economic reforms ", The European Journal of the History of Economic Thought, $\mathrm{n}^{\mathrm{o}} 1-3,1994$, p. 519-550. p. $57-74$.

(48) Ernest Lluch, El pensament econòmic a Catalunya (1760-1840), Barcelone, 1973,

(49) Jesús Astigarraga, «Estudio Preliminar», Ilustración y economía en Navarra (1770-1793), Vitoria, 1996, p. LIX-LXXII. 
En 1781, elles furent utilisées comme critère d'autorité dans les débats des Cortès de Navarre afin de contrer un projet de loi qui, au nom d'arguments agraristes et physiocrates, prétendait libérer de son carcan régulateur le commerce des grains. En 1789, un influent auteur des Lumières navarraises, Magallón, plagia les idées de Necker sur la propriété privée et les responsabilités de l'administration quant à l'approvisionnement en grains afin de justifier un programme interventionniste de réorganisation du système des halles publiques destiné à résoudre la forte crise de subsistances que traversait alors la Navarre.

Sur la législation fit l'objet d'un dernier plagiat de la part de l'économiste aragonais Anzano, fonctionnaire aguerri de l'administration bourbonienne ${ }^{50}$. Ce plagiat était inséré dans sa traduction de l'Essai sur la police générale des grains (1753) de Herbert, publiée en 1795. Loin de se borner à exposer les idées optimistes et libérales de cet illustre héritier de Gournay, Anzano ajoutait à sa traduction une longue série d' « observations » personnelles; celles-ci se trouvaient à la suite de chacun des vingt chapitres du l'ouvrage, composant ainsi une sorte de « livre dans le livre ». Dans ces « observations », Anzano se livrait à une révision très critique des idées de Herbert, y opposant systématiquement les propositions modérées et interventionnistes de Necker et recommandant d'utiliser en Espagne les instruments concrets dont celui-ci s'était servi pour réguler le marché des grains. Le commanditaire de cette traduction critique n'était autre que le Conseil de Castille, le principal organe politique de la monarchie, ce qui permet de voir que ses influents conseillers considéraient l'œuvre de Necker comme particulièrement appropriée pour canaliser le développement agraire en Espagne à une époque où, en raison du changement de cycle agricole et d'une mauvaise conjoncture économique, le rythme des réformes libérales que la politique agraire officielle avait lancées avec la Pragmática de 1765 connaissait un fort ralentissement.

Une autre preuve importante de l'impact de Sur la législation sur les autorités centrales des Lumières espagnoles est que le livre influença la littérature économique relative à l'élaboration du dossier sur la Loi Agraire (Expediente de la Ley Agraria). Ce dossier suscita, au long des années 1760-1795, un grand nombre de réflexions économiques dont l'expression la plus significative est le fameux Informe de Ley Agraria de

(50) Cf. Jesús Astigarraga et Javier Usoz, «Política y Economía en el Análisis del Comercio del Trigo (1795) de Tomás Anzano », Hispania, nº 232, mai-août 2009, p. 395-421. 
Jovellanos, présenté en 1795 à la Société de Madrid. Ce rapport est considéré comme l'un des textes les plus aboutis du libéralisme des Lumières espagnol. Des études récentes ont mis en évidence dans ce texte la place importante des idées de Necker dont Jovellanos était un lecteur assidu. Son analyse sur le rôle de l'opinion et des comportements psychologiques dans le fonctionnement du marché des grains, comme les thèses qui soustendent l'opposition à la libre exportation des grains, procèdent de Sur la législation $^{51}$. Quoi qu'il ne citât pas sa source, Jovellanos en venait même à plagier la proposition de loi faite par Necker en vue de réguler ce secteur du commerce en France. Tout cela ne fait qu'attester que Sur la législation fut un texte central en Espagne dans les vingt dernières années du $\mathrm{XVIII}^{\mathrm{e}}$ siècle et qu'il fut utilisé par les auteurs et les acteurs des Lumières tant pour affiner leur analyse du fonctionnement du marché des grains que pour en favoriser la réforme ${ }^{52}$.

\section{L'administration territoriale}

La troisième œuvre de Necker traduite en Espagne fut Des administrations provinciales. Necker avait consacré ce mémoire, publié en 1781, à l'exposition de son principal projet de décentralisation politique et économique du royaume, ainsi qu'à expliquer les lignes directrices de son programme expérimental de création des administrations provinciales du Berry (1778) et de la Haute-Guyenne (1779). Cinq ans après parut la traduction espagnole du mémoire, dont l'auteur était Torre y Mollinedo, membre de la Société des Amis du Pays de Madrid. Les vues de ce dernier, en réalisant cette version, étaient directement en rapport avec son statut de haut fonctionnaire des Finances (Real Hacienda) où il occupait une charge d'officier chargé des comptes (Oficial Mayor de la Contaduría del Cargo de la Superintendencia General de Juros). Comme nous allons le voir, sa traduction avait un caractère éminemment « politique », car elle

(51) Cf. Jesús Astigarraga, « Necker y Jovellanos : un “área neckeriana” en el Informe de Ley Agraria », Revista de Historia Económica, n XVII-2, 1998, p. 559-570.

(52) D'autres auteurs qui adaptèrent les idées de Sur la législation à la réalité agraire espagnole furent Villava, Generés, Calomarde, Asso, Zuaznabar et Cabarrús. Un certain parallélisme peut être noté entre ce dernier et Necker, les deux partageant la condition de grands financiers, d'hommes politiques et de penseurs des Lumières. Cette ressemblance s'accentue si l'on songe, comme en témoigne un document référencé dans le catalogue de l'Archivo Histórico Nacional, que l'économiste de Bayonne tenta en 1790 de remplacer Necker à la tête du ministère des Finances. Néanmoins, de par ses sympathies physiocrates et son radicalisme fiscal, Cabarrús se montra toujours très critique envers Necker. Sur leurs relations, voir Michel ZyLBERBERG, Une si douce domination, Paris, 1993. 
se rapportait à la réforme de l'administration territoriale lancée à l'époque par le ministre des Finances Lerena.

Lorsque Torre entreprit cette version, il avait derrière lui vingt années d'expérience de traductions et d'écrits sur des sujets économiques. Une grande partie de son œuvre demeure toutefois inédite et n'a toujours pas fait l'objet d'une étude systématique. Nous avons ici affaire à un fonctionnaire zélé de second rang dont les écrits furent réalisés sous la protection de personnalités comme Lerena ou le comte de Aranda, président du Conseil de Castille. Ses textes présentent des caractéristiques très marquées : en premier lieu, tous reflètent une réflexion approfondie sur la règlementation et les finances, exprimée sous forme de manuels pour la formation des officiers des Finances. Le second trait très caractéristique de ces écrits est leur orientation industrialiste : Torre était un défenseur acharné de la promotion des manufactures et des corps de métiers. Tout porte donc à croire que sa principale référence doctrinale fut la science de la police. De fait, outre la traduction de Necker, son principal apport aux Lumières espagnoles fut la traduction, entre 1767 et 1801, des Institutions politiques (1760) du caméraliste Bielfeld, auteur dont il ne fut pas seulement le traducteur mais un grand prosélyte et divulgateur des idées ${ }^{53}$.

La traduction de Torre présentait trois traits remarquables ${ }^{54}$. D'une part, il s'agissait d'une version de grande qualité, quoique légèrement résumée; de l'autre, elle s'ouvrait sur une dédicace à Lerena, qui avait sans doute commandité la dite traduction. Torre établissait un certain parallélisme entre Necker et le ministre des Finances espagnol, dont il faisait l'éloge de la trajectoire politique. Il louait en particulier le sens de la justice et de l'équité qui avait inspiré sa réforme récente des rentas provinciales. La troisième caractéristique était les vingt-deux notes personnelles dont le traducteur enrichissait le texte : dépourvues de toute réflexion originale, celles-ci provenaient presque systématiquement de Des finances et répondaient à trois objectifs précis. Il s'agissait tout d'abord de comprendre la nature des différentes institutions économiques, administratives et politiques françaises; bon nombre d'entre elles visaient à exposer en détail les diverses réalités fiscales. En second lieu, grâce à ces notes, Torre tentait de familiariser le lecteur avec les expériences d'administrations pro-

(53) Ernest Lluch, Las España vencidas del Siglo XVIII, Barcelone, 1999, p. 146-148.

(54) Memoria reservada sobre el establecimiento de rentas provinciales en un pie ventajoso al público y al Estado que trabajó y presentó Mr. Necker, Madrid, 1786. Le Memorial literario (novembre 1786, p. 338) consacra un compte rendu à cet ouvrage de Necker. 
vinciales tentées avec des «avantages manifestes » dans le Berry et la Haute-Guyenne ${ }^{55}$. En dernier lieu, il présentait la voie française comme convenant à la réalité espagnole : les idées de Necker méritaient « l'attention la plus scrupuleuse de tout chef du département des Finances dès lors que celui-ci envisage d'imposer et d'exiger des communautés qu'elles payent un tribut $»^{56}$.

Comme nous l'avons dit, la traduction de Torre avait clairement une visée politique et c'est certainement la raison pour laquelle ce livre de Necker, l'un des moins traduits dans le cadre européen, fit rapidement son entrée dans les Lumières espagnoles. Cela se vérifie encore plus si l'on considère qu'eut lieu en Espagne, au cours des années 1780, une décentralisation de l'administration territoriale à laquelle l'exemple des administrations provinciales de Necker ne fut pas étrangère. La première initiative date de 1780 et fut préparée en sous-main par Floridablanca et Múzquiz. Tous deux décidèrent que de nouvelles Juntes provinciales seraient fondées dans les capitales des provinces de la couronne de Castille. Ces Juntes possédaient deux traits clairement «neckeriens » : leur caractère collégial et leur motivation fiscale. Les ministres entendaient remplacer la gestion traditionnelle des intendants, délégués du pouvoir royal dans les provinces, par une structure collégiale, pourvue de fonctions fiscales et statistiques, en plus de ses compétences générales sur le développement de l'économie locale. Toutefois, cette innovation ne faisait que préparer une réforme qui devait s'appliquer dans des conditions politiques plus favorables - une fois que la guerre avec l'Angleterre aurait pris fin -, de sorte que sa mise en place allait revenir à Lerena. Celui-ci, afin de rendre plus efficace sa réforme des rentas provinciales, profita de la traduction réalisée par son subordonné Torre pour promulguer en 1787 un important « règlement sur l'intervention de l'administration », dans lequel était remise au goût du jour l'idée de fonder des Juntes provinciales. Dans ce règlement, il était établi que le contrôle des Juntes passerait aux mains des comptables qui furent élevés à la catégorie de Fiscales Generales de Rentas au détriment des intendants. La mesure visait à miner le pouvoir traditionnel de ces derniers dans l'administration locale, du fait qu'ils se trouvaient constamment suspectés de faire un usage arbitraire de leurs compétences très étendues. De plus, si la fonction principale des Juntes provinciales consistait toujours à assurer « la meilleure administration,

(55) Ibid., note VI, p. 8-9. Torre mentionnait aussi d'autres tentatives ayant été vouées à l'échec (par exemple le cas du Dauphiné).

(56) Ibid., note VIII, p. 13. 
perception et gouvernement » des rentas provinciales et autres impôts, elles furent également chargées d'intervenir dans le champ de la justice, de l'armée - y compris pour lever des troupes - et du développement de l'économie et de la démographie des provinces, ainsi que de leurs études statistiques. Fidèle aux principes de Necker, Lerena favorisa l'établissement de ces Juntes, à partir de 1787, sur le mode expérimental et selon un principe d'application graduel et progressif ${ }^{57}$.

En dépit de son indéniable ambition décentralisatrice, le nouveau système de Lerena ne possédait pas la même profondeur que son modèle français. En premier lieu, les Juntes provinciales étaient conçues comme de simples organes administratifs surbordonnés à la direction des Finances et ne furent jamais considérées comme d'authentiques administrations provinciales, avec une structure politique représentative des trois « états » et de larges compétences économiques, comme dans le cas des assemblées françaises du Berry et de la Haute-Guyenne. En second lieu, en dépit de sa modération, le programme de Lerena vint allonger la liste de ces nombreux projets sans suite élaborés par les hommes des Lumières. Les résistances locales, les critiques des secteurs conservateurs sur l'incompatibilité des Juntes provinciales avec l'ordre monarchique et le manque d'initiative du ministère des Finances qui, non content de ne pas définir avec précision leurs compétences, craignait que leur création n'entraînât une augmentation du nombre de fonctionnaires, firent que les Juntes n'allèrent jamais au-delà du stade expérimental, bien qu'elles aient existé jusqu'en 1802.

Toutefois, l'influence de Des administrations proviciales en Espagne ne se limita pas à cette expérience infructueuse de Lerena. D'autres expériences de décentralisation tentées dans l'espace européen étaient très connues en Espagne; cela encouragea un courant d'opinion qui, depuis plusieurs années, réclamait un accroissement du pouvoir des organes locaux, ainsi qu'une modernisation de leur organisation ${ }^{58}$. Ce courant décentralisateur connut au cours des années 1780 un intéressant processus de rénovation, encouragé par la connaissance non seulement du modèle de Necker, mais aussi des positions plus radicales défendues par Turgot-Dupont de Nemours dans le Mémoire sur les municipalités ${ }^{59}$. De leur côté, les économistes les plus clairement «neckeriens »

(57) La première tentative eut lieu dans les provinces de La Mancha, Guadalajara, Ávila y Cuenca.

(58) Ernest Lluch, Las España vencidas, op. cit., p. 129-162.

(59) Cf. Javier Fernández Sebastián, La Ilustración política, Bilbao, 1994, p. 98-102, et Pablo Fernández Albadejo, op. cit., p. 480. 
- Torre, Almodóvar, Alcalá et Mantecón - s'efforcèrent de démontrer que l'Espagne devait se limiter à « reproduire» les administrations provinciales françaises ${ }^{60}$. Leurs arguments reprenaient littéralement les idées exposées dans Des administrations provinciales mais puisaient aussi à d'autres sources telles que Mirabeau, Montesquieu et plus encore le physiocrate suisse Schmid d'Avenstein et le ministre prussien Hertzberg.

Par ailleurs, si leurs réclamations avaient un ton politique modéré, elles n'étaient pas pour autant négligeables dans le contexte du débat constitutionnel que l'Espagne des Lumières avait lancé aux alentours de 1780. L'une des principales innovations que proposaient Alcalá et Mantecón était précisément la défense d'institutions représentatives dotées de fonctions exécutives, qu'ils considéraient compatibles avec le système monarchique, contrairement aux secteurs conservateurs. Cela leur permit de mettre en cause pour la première fois l'utilité des Sociétés économiques des Amis du Pays qui étaient le fondement institutionnel de la principale expérience de décentralisation économique entreprise à partir de 1765 sous le règne de Charles III. Les deux auteurs qui, comme on l'a vu, étaient des membres actifs de la Société de Ségovie, en arrivaient à soutenir que la constitution de ces sociétés était " défectueuse », en raison de leur régime d'association ouverte, le nombre indéfini de membres et leur caractère peu représentatif : cela les amenait à réclamer leur transformation en administrations provinciales. Celles-ci étaient censées absorber les fonctions des Sociétés économiques en conseillant le gouvernement, en surveillant la situation économique des provinces et en menant à bien les réformes nécessaires. Fidèles à leur vision gradualiste, ils suggéraient de commencer par établir, «à titre d'essai », une seule administration provinciale à laquelle serait confiée la réforme des finances sur le plan local. Celle-ci amènerait de nombreux avantages car, une fois de plus en accord avec Necker, elle permettrait de mettre au point « une méthode constante, équitable et adaptée aux circonstances particulières de chaque province dans les Finances royales ».

Avec ces commentaires, Alcalá et Mantecón se faisaient probablement l'écho d'un état de l'opinion bien plus général et d'une problématique plus strictement politique car ils coïncidaient chronologiquement avec les points de vue du duc d'Almodóvar. Cet influent diplomate fut l'un des principaux promoteurs du système politique britannique et des expé-

(60) Duc D’Almodóvar, op. cit., p. 37-47; Vicente Alcalá et Vicente Mantecón, op. cit., 
riences de décentralisation de Necker dans l'Espagne des années 1780. Il percevait certaines similitudes entre les Sociétés économiques espagnoles et les administrations provinciales françaises et il défendait l'implantation de ces dernières en Espagne en raison du « nouveau lustre » qu'acquerrait grâce à elles le « droit de représentation parlementaire ${ }^{61}$. Ainsi, tout porte à croire que, dans les premiers moments du débat constitutionnel qui allaient conduire aux Cortes de Cádiz, les écrits de Necker offraient comme avantage les positions philo-britanniques reconnues et favorables à la monarchie constitutionnelle de leur auteur. Pourtant, aucune des idées proposées par Almodóvar, Mantecón ou Alcalá ne connut d'effet pratique : il n'y eut pas en Espagne de réforme profonde des Sociétés économiques et encore moins de création d'administrations provinciales. Moyennant quoi, des libéraux espagnols du $\mathrm{XIX}^{\mathrm{e}}$ siècle parmi les plus influents continuèrent à considérer que celles-ci étaient nécessaires à une organisation correcte de l'administration : en 1834, le ministre des Finances et économiste libéral Canga Argüelles en venait à copier de nouveau littéralement, quoique sans les citer, de longs passages de Des administrations provinciales pour justifier le fait qu'il convenait de fonder en Espagne des Juntes provinciales administratives ${ }^{62}$.

Il exista donc au sein des Lumières économiques espagnoles une littérature d'inspiration "neckerienne » composée des traductions des textes de l'économiste genevois et d'un vaste ensemble de plagiats, d'adaptations, de compte rendus et de résumés de ses écrits, parmi lesquels ressortent les travaux de Caresmar, Alcalá, Mantecón, Magallón, Almodóvar et Anzano. Au total, entre 1780 et 1795, les auteurs espagnols des Lumières publièrent ou tentèrent de publier dix versions des écrits de Necker : six traductions, trois plagiats-adaptations et un résumé. Le Genevois fut donc peut-être l'économiste le plus traduit de tout le $\mathrm{XVIII}^{\mathrm{e}}$ siècle en Espagne. Ce recours à la traduction fut accompagné de

(61) Duc D’Almodóvar, op. cit., p. 41 et 46. Celui-ci était depuis 1785 Directeur de la Société Économique de Ségovie et, n'en était pas moins très lié avec Alcalá et Mantecón.

(62) José CAnga ArgüElles, op. cit., vol. II, p. 27-29. La figure politique de Necker allait réapparaître en Espagne sous le Trienio liberal (1820-1823), grâce à la traduction espagnole d'un opuscule publié de manière anonyme en 1789 et attribué à A.-J.-M. SEVAN, Conversación del Señor Necker con la Condesa de Polignac, el Barón de Breteuil y el Abad de Bermond, traducida del francés por el ciudadano A. H. M., Madrid, 1820. Cette persistance de la référence à Necker se retrouve aussi chez des auteurs comme Soto, Torrente, Valle ou Colmeiro, c'est-à-dire au moins jusqu'aux années 1840 . 
nombreuses informations sur Necker et son œuvre qui paraissaient dans les publications périodiques espagnoles.

Par rapport à l'ensemble du cadre européen, le cas espagnol présente trois particularités notables: un relatif décalage chronologique, l'existence d'une traduction de Des administrations provinciales - œuvre dont la diffusion internationale fut plutôt rare - et l'absence, au contraire, de l'un des ouvrages les plus répandus de Necker, Des Finances. Néanmoins, celui-ci fut le plus influent sur le long terme; il continua à être consulté par les économistes et les financiers espagnols des premières années du XIX ${ }^{\mathrm{e}}$ siècle, ce qui constitue un curieux cas d'asymétrie entre traduction et influence effective. Une bonne partie des traductions et des adaptations de Necker en Espagne avaient une importante connotation politique, au sens où elles furent élaborées sous la protection de puissantes personnalités - voire directement commandées par celles-ci - pour être utilisées dans les réformes de la période en matière d'économie politique, marquées par l'esprit des Lumières. De fait, le courant «neckerien » eut une influence très notable dans la politique économique de l'époque, notamment dans le domaine des finances publiques, du marché des grains et de l'administration territoriale. En outre, l'œuvre de Necker favorisa l'apparition progressive d'une « opinion publique ».

Ce formidable succès espagnol de Necker ne peut s'expliquer uniquement par la conception traditionnelle qui considère ses écrits comme une simple réaction contre la physiocratie et son libéralisme radical et dogmatique. Il est nécessaire d'y apporter un nouveau point de vue, prenant en compte d'autres questions essentielles. En premier lieu, il faut considérer l'avantage comparatif qu'offrait aux Espagnols des Lumières une œuvre comme celle de Necker, favorable à un libéralisme économique modéré et à un réformisme progressif et pragmatique qui, quoi qu'on en ait pu dire, ne manquait ni de sincérité ni de profondeur et dont la viabilité pratique avait été largement démontrée lors de son ministère. En second lieu entre en ligne de compte son orientation politique, proche du modèle britannique et favorable à la monarchie constitutionnelle, qui fut invoquée dans le cadre du débat constitutionnel par les secteurs favorables à des réformes modérées. En lien avec cela figure, en troisième lieu, l'option décentralisatrice, sur le plan politique comme sur le plan administratif, qui contribuait à ranimer la structure de la monarchie et connut un accueil remarquable au sein des Lumières espagnoles. Enfin, il convient de prendre en compte l'affinité de ses idées économiques avec le noyau des auteurs européens du " système intermédiaire » dont l'influence dominante dans la pensée économique espagnole des Lumières est aujourd'hui 
fermement attestée. De par son caractère pragmatique et relativiste et sa tonalité modérée en matière économique et politique, la pensée de Necker avait donc toutes les chances de convenir à des Lumières aussi tamisées que les Lumières espagnoles.

Jesús Astigarraga

Université de Saragosse Département de Structure et d'Histoire Économique et d'Économie Publique Faculté de Droit, c/Pedro Cerbuna, 12, 50009 Saragosse - Espagne astigarr@unizar.es 